\title{
Effect of Robot-Assisted Game Training on Upper Extremity Function in Stroke Patients
}

\author{
Kyeong Woo Lee, MD, Sang Beom Kim, MD, Jong Hwa Lee, MD, Sook Joung Lee, MD, Jin Wan Kim, MD
}

Department of Physical Medicine and Rehabilitation and Regional Cardiocerebrovascular Center, Dong-A University College of Medicine, Busan, Korea

\begin{abstract}
Objective To determine the effects of combining robot-assisted game training with conventional upper extremity rehabilitation training (RCT) on motor and daily functions in comparison with conventional upper extremity rehabilitation training (OCT) in stroke patients.

Methods Subjects were eligible if they were able to perform the robot-assisted game training and were divided randomly into a RCT and an OCT group. The RCT group performed one daily session of 30 minutes of robotassisted game training with a rehabilitation robot, plus one daily session of 30 minutes of conventional rehabilitation training, 5 days a week for 2 weeks. The OCT group performed two daily sessions of 30 minutes of conventional rehabilitation training. The effects of training were measured by a Manual Function Test (MFT), Manual Muscle Test (MMT), Korean version of the Modified Barthel Index (K-MBI) and a questionnaire about satisfaction with training. These measurements were taken before and after the 2 -week training.

Results Both groups contained 25 subjects. After training, both groups showed significant improvements in motor and daily functions measured by MFT, MMT, and K-MBI compared to the baseline. Both groups demonstrated similar training effects, except motor power of wrist flexion. Patients in the RCT group were more satisfied than those in the OCT group.

Conclusion There were no significant differences in changes in most of the motor and daily functions between the two types of training. However, patients in the RCT group were more satisfied than those in the OCT group. Therefore, RCT could be a useful upper extremity rehabilitation training method.
\end{abstract}

Keywords Stroke, Robotics, Video games, Upper extremity, Rehabilitation

\section{INTRODUCTION}

A stroke is a central nervous system disease caused by cerebrovascular problems such as infarction or hemorrhage. Stroke may lead to impairment of various physical functions, including hemiplegia, language disorder,

Received July 22, 2016; Accepted October 10, 2016

Corresponding author: Jin Wan Kim

Department of Physical Medicine and Rehabilitation \& Regional Cardiocerebrovascular Center, Dong-A University College of Medicine, 26 Daesingongwon-ro, Seo-gu, Busan 49201, Korea. Tel: +82-51-240-5690, Fax: +82-51-254-8511, E-mail: tiyon@naver.com

ORCID: Kyeong Woo Lee (http://orcid.org/0000-0001-8785-5535); Sang Beom Kim (http://orcid.org/0000-0002-5622-5933); Jong Hwa Lee (http:// orcid.org/0000-0003-2489-358X); Sook Joung Lee (http://orcid.org/0000-0002-6894-445X); Jin Wan Kim (http://orcid.org/0000-0002-8240-6332).

(c) This is an open-access article distributed under the terms of the Creative Commons Attribution Non-Commercial License (http://creativecommons.org/ licenses/by-nc/4.0) which permits unrestricted noncommercial use, distribution, and reproduction in any medium, provided the original work is properly cited. Copyright (c) 2017 by Korean Academy of Rehabilitation Medicine 
swallowing disorder or cognitive disorder, according to the location and degree of morbidity [1]. Among these, hemiplegia is a common symptom occurring in $85 \%$ of stroke patients. In particular, upper extremity paralysis is more frequent and requires longer recovery time than lower extremity paralysis $[2,3]$. To maintain the basic functions of ordinary life, the use of the upper extremities is essential; therefore, upper extremity paralysis commonly causes problems in performing the activities of daily living [2].

Robot-assisted rehabilitation treatment has recently been widely investigated as an effective neurorehabilitation approach that may augment the effects of physical therapy and facilitate motor recovery [4]. Robot-assisted rehabilitation treatments have been developed in recent decades to reduce the expenditure of therapists' effort and time, to reproduce accurate repetitive motions and to interact with force feedback $[5,6]$. The most important advantage of using robot-assisted rehabilitation treatment is the ability to deliver high-dosage and high-intensity training [7].

In rehabilitation patients may find such exercises monotonous and boring, and may lose motivation over time [8]. Upper extremity rehabilitation training using video games, such as Nintendo Wii games and the PlayStation EyeToy games, enhanced upper extremity functions and resulted in greater patient satisfaction than conventional rehabilitation treatment [9-13].

The objective of this study was to determine the effects of combining robot-assisted game training with conventional upper extremity rehabilitation training (RCT) on motor and daily functions in comparison to conventional upper extremity rehabilitation training (OCT) in stroke patients. This study was a randomized controlled trial and we evaluated motor power, upper extremity motor function, daily function and satisfaction.

\section{MATERIALS AND METHODS}

\section{Subjects}

The subjects of this study were acute and sub-acute phase stroke patients hospitalized in Dong-A University Hospital from April 2014 to January 2016. In accordance with previous studies and accepted standards for conducting robot-assisted game training, patients who were able to undertake robot-assisted game training, maintain a sitting posture stably on a chair, have scores of 2 points or higher on the Manual Muscle Test (MMT) in adductor and abductor of the shoulder joints and flexor and extensor of the elbow joints, and a score 10 or higher on the Korean version of the Mini-Mental Status Examination (K-MMSE) were included [9,14-16]. Those who had a previous history of stroke or neuromuscular disorder, trauma on the upper limbs or the upper chest area, or anamnesis, such as a history of surgery, were excluded.

The Institutional Review Board of Dong-A University Hospital approved the study (No. 16-121), and written consent was received from all patients.

\section{Methods}

Enrolled patients who met the inclusion criteria were randomly selected and assigned to the RCT group or the OCT group.

The RCT group performed one daily session consisting of 30 minutes of robot-assisted game training using the upper extremity rehabilitation robot Neuro-X (Apsun Inc., Seoul, Korea) and one daily session of 30 minutes of conventional upper extremity rehabilitation training, 5 days a week for 2 weeks. The OCT group performed two daily sessions of 30 minutes of conventional upper extremity rehabilitation training. Each training protocol was applied over 20 sessions (30 minutes per session, 2 sessions per day, 5 days a week for 2 weeks).

\section{Robot-assisted game training programs}

The robot-assisted game training was delivered using the upper extremity rehabilitation robot Neuro-X. Neuro$\mathrm{X}$ is composed of a video monitor, a robot arm and a computer. The length of the robot arm is adjustable to be suitable for a patient (Fig. 1). This device is a two-degreeof-freedom robot, allowing patients to perform shoulder abduction-adduction and elbow flexion-extension movements in the horizontal plane. This device has several treatment modes, including continuous passive motion exercise (CPM) mode, controlled assisted movement exercise (CAM, or active assisted exercise) mode, and game mode. The patient, in a sitting posture, has the paretic side arm fixed onto the robot arm. The patient moves the robot arm with abduction and adduction of the shoulder joints, as well as with flexion and extension of the elbow joints, to perform movements in each game program. The wrist joint was fixed and was not used for the game 
program (Fig. 1). For the robot-assisted game training, the Arkanoid game, car driving game, jumping rope game, dolphin \& circus game, goalkeeper game and fruit target game were used. Subjects were allowed to select a game freely according to their preference (Fig. 2). When the first session was started, a therapist taught the patient
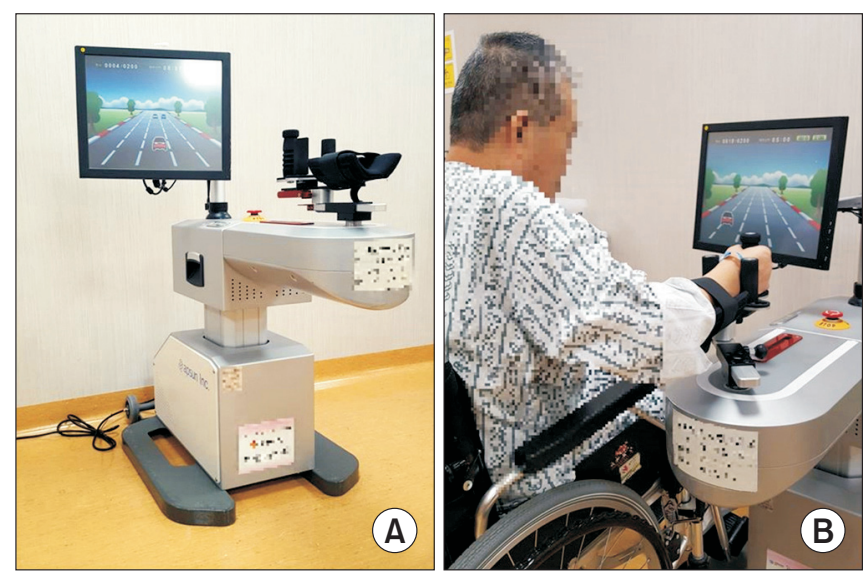

Fig. 1. (A) Neuro-X, an upper extremity rehabilitation robot, consisting of a video monitor, a robot arm and a computer. (B) The patient performing robot-assisted game training with the upper extremity rehabilitation robot. how to play the game and demonstrated game play for about 15 minutes. During training, a therapist supervised the patient's shoulder joint and elbow joint in order to keep the joint positioned safely. If needed, the patients' trunk was restrained by a harness to decrease compensatory movements.

\section{Conventional upper extremity rehabilitation training \\ Conventional upper extremity rehabilitation training consisted of a muscle stretching exercise, a joint exercise, a muscle strengthening exercise and training in the ac- tivities of daily living. The training was performed by the occupational therapists.}

\section{Evaluation methods}

Basic information on stroke patients, i.e., age, gender, type of lesion, location of the lesion, side of paresis, duration of disease, K-MMSE and Beck Depression Inventory (BDI) were investigated. Values for MMT, Manual Function Test (MFT) and Korean version of the Modified Barthel Index (K-MBI) were measured for both groups before and after training. To evaluate training effects, changes of MMT scores, MFT scores and K-MBI scores were calculated from baseline to end of 2-week training. After the
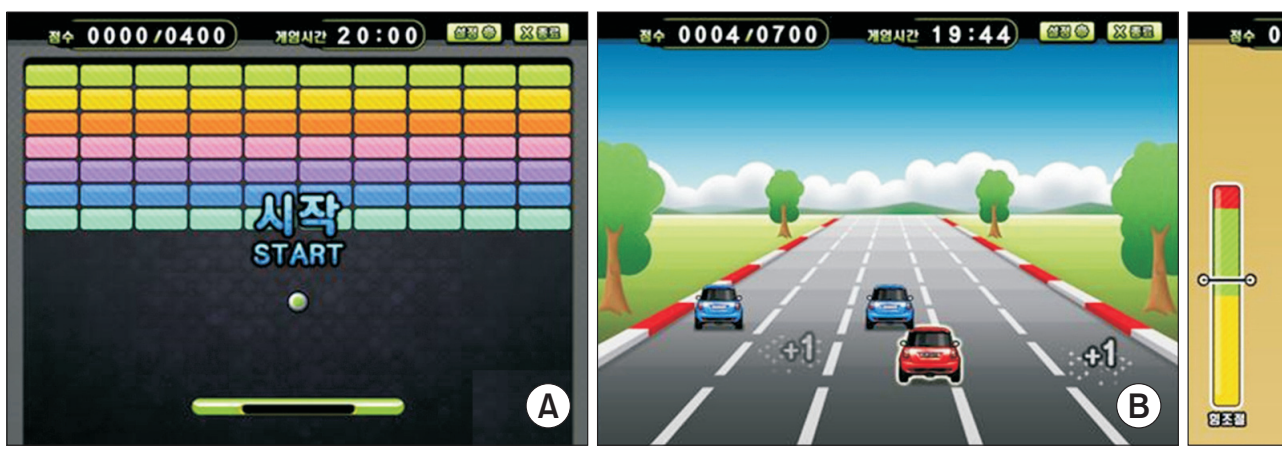

$0002 / 1400$
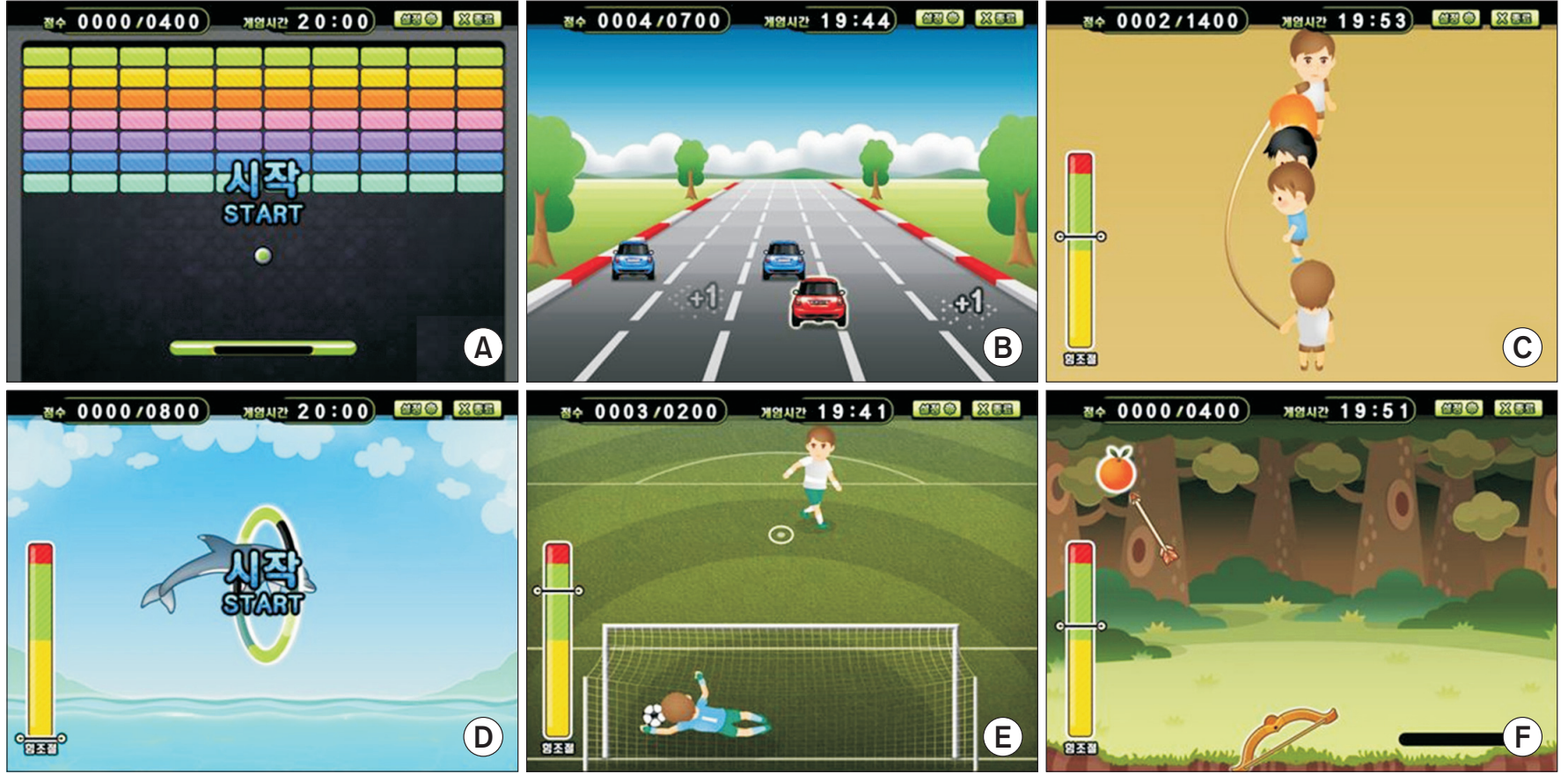

Fig. 2. Six game programs on the Neuro-X (Apsun Inc., Seoul, Korea). (A) Arkanoid game. (B) Car driving game. (C) Jumping rope game. (D) Dolphin \& circus game. (E) Goalkeeper game. (F) Fruit target game. 
training, a survey on side- effects and degree of satisfaction was conducted.

MMT was performed on adduction and abduction of the shoulder joints, as well as on flexion and extension of the elbow joints, flexion and extension of the wrist joints, and hand grip motion. Each motion was evaluated by six steps according to the accepted methods for measuring manual muscle strength. Upper extremity motor functions were evaluated using the MFT. This test comprised eight items: shoulder joint flexion, abduction, external rotation, internal rotation, whether the subject was able to grasp a ball or pick it up, the number of regular $4 \mathrm{~cm}$ hexahedron blocks moved within 5 seconds and $30 \mathrm{sec}-$ onds. The total possible score was 32 points [17]. In addition, K-MBI was used to evaluate the patients' activities of daily living. A survey on their degree of satisfaction with the training was conducted in both groups after training was completed. Patients also described side-effects of the training. Their degree of satisfaction with the training was recorded on a scale of 1 to 5 (very satisfied $=5$ points, satisfied $=4$ points, moderately satisfied $=3$ points, dissatisfied $=2$ points, and very dissatisfied $=1$ point).

\section{Statistical analysis}

The Korean version of Windows SPSS 18.0 (SPSS Inc., Chicago, IL, USA) was employed. The Wilcoxon signedrank test was carried out to evaluate MMT, MFT, and KMBI before and after training. For comparison between the two groups, statistical processing was conducted with the Mann-Whitney U-test. A statistical significance level was set at $\mathrm{p}<0.05$.

\section{RESULTS}

Three hundred forty-five stroke patients had been admitted to our department, of which 58 patients were enrolled in our study. Twenty-nine patients each were assigned randomly to the RCT or the OCT group. Eight patients dropped out due to early discharges from the hospital and declines in medical conditions. Finally, 50 subjects were included and completed this study (Fig. 3). There were no significant differences between the two groups in their average age, lesion type, lesion location, paretic side, duration of disease, K-MMSE, or BDI scores at baseline (Table 1). The adduction and abduction of the shoulder joints, flexion and extension of the elbow joints, flexion and extension of the wrist joints, and hand grip motion did not significantly differ between the two groups in MMT, MFT, and K-MBI at baseline assessment (Table 1).

Both groups had significant improvements in the adduction and abduction of the shoulder joints, flexion and extension of the elbow joints, flexion and extension of the wrist joints, and hand grip motion in MMT, MFT, and KMBI after the 2-week training compared to before training $(\mathrm{p}<0.05)$ (Table 2).

When the two groups' training effects were compared, there were no significant differences between the two

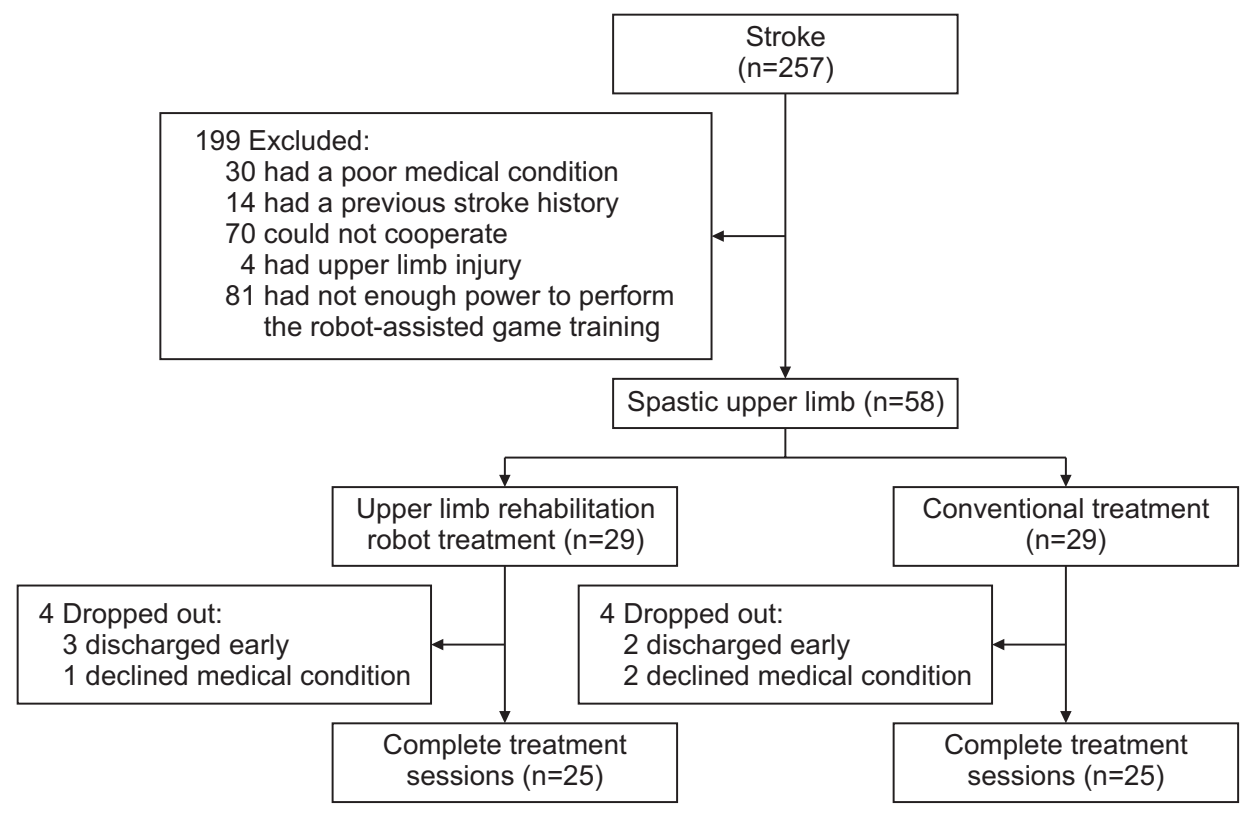

Fig. 3. The algorithm for enrollment of the subjects. 
Table 1. Baseline characteristics

\begin{tabular}{|lccc|}
\hline & RCT $(\mathbf{n}=\mathbf{2 5})$ & OCT (n=25) & p-value \\
\hline Age & $55.76 \pm 13.60$ & $57.88 \pm 11.12$ & 0.547 \\
\hline Sex (male:female) & $14: 11$ & $12: 13$ & \\
\hline Lesion & $17: 8$ & & \\
\hline Ischemic:hemorrhagic & $16: 9$ & $15: 10$ & \\
\hline Cortical:subcortical & $12: 13$ & $11: 14$ & 0.733 \\
\hline Paretic side (right:left) & $15.40 \pm 8.05$ & $14.40 \pm 6.95$ & 0.741 \\
\hline Days from stroke onset & $22.72 \pm 4.43$ & $22.32 \pm 4.44$ & 0.590 \\
\hline K-MMSE & $11.96 \pm 4.69$ & $12.36 \pm 3.08$ & \\
\hline BDI & & & 0.463 \\
\hline MMT & $2.52 \pm 0.71$ & $2.36 \pm 0.57$ & 0.328 \\
\hline Shoulder adduction & $2.60 \pm 0.76$ & $2.40 \pm 0.50$ & 0.475 \\
\hline Shoulder abduction & $2.04 \pm 0.84$ & $2.16 \pm 0.55$ & 0.776 \\
\hline Elbow flexion & $2.12 \pm 0.73$ & $2.08 \pm 0.57$ & 0.693 \\
\hline Elbow extension & $1.32 \pm 0.56$ & $1.24 \pm 0.44$ & 0.951 \\
\hline Wrist flexion & $1.20 \pm 0.50$ & $1.16 \pm 0.37$ & 0.641 \\
\hline Wrist extension & $1.12 \pm 0.33$ & $1.08 \pm 0.28$ & 0.486 \\
\hline Grasp & $8.80 \pm 2.35$ & $8.72 \pm 3.35$ & 0.611 \\
\hline MFT & $47.56 \pm 6.63$ & $46.36 \pm 13.82$ & \\
\hline K-MBI & & \\
\hline
\end{tabular}

Values are presented as mean \pm standard deviation or number.

RCT, combining robot-assisted game training with conventional upper extremity rehabilitation training; OCT, only conventional upper extremity rehabilitation training; K-MMSE, Korean version of Mini-Mental Status Examination; BDI, Beck Depression Inventory; MMT, Manual Muscle Test; MFT, Manual Function Test; K-MBI, Korean version of Modified Barthel Index.

Table 2. Changes of measurements after training

\begin{tabular}{|c|c|c|c|c|c|c|}
\hline & \multicolumn{2}{|c|}{ RCT $(n=25)$} & \multirow{2}{*}{ p-value } & \multicolumn{2}{|c|}{ OCT $(n=25)$} & \multirow{2}{*}{ p-value } \\
\hline & Pre & Post & & Pre & Post & \\
\hline \multicolumn{7}{|l|}{ MMT } \\
\hline Shoulder adduction & $2.52 \pm 0.71$ & $2.96 \pm 0.79$ & $0.009^{*}$ & $2.36 \pm 0.57$ & $2.84 \pm 0.55$ & $0.001^{*}$ \\
\hline Shoulder abduction & $2.60 \pm 0.76$ & $3.08 \pm 0.70$ & $0.003^{*}$ & $2.40 \pm 0.50$ & $2.92 \pm 0.57$ & $0.000^{*}$ \\
\hline Elbow flexion & $2.04 \pm 0.84$ & $2.80 \pm 0.87$ & $0.001^{*}$ & $2.16 \pm 0.55$ & $2.72 \pm 0.61$ & $0.000^{*}$ \\
\hline Elbow extension & $2.12 \pm 0.73$ & $2.64 \pm 0.81$ & $0.003^{*}$ & $2.08 \pm 0.57$ & $2.56 \pm 0.71$ & $0.003^{*}$ \\
\hline Wrist flexion & $1.32 \pm 0.56$ & $1.72 \pm 0.61$ & $0.046^{*}$ & $1.24 \pm 0.44$ & $2.12 \pm 0.67$ & $0.000^{*}$ \\
\hline Wrist extension & $1.20 \pm 0.50$ & $1.72 \pm 0.61$ & $0.003^{*}$ & $1.16 \pm 0.37$ & $1.68 \pm 0.56$ & $0.000^{*}$ \\
\hline Grasp & $1.12 \pm 0.33$ & $1.56 \pm 0.58$ & $0.005^{*}$ & $1.08 \pm 0.28$ & $1.56 \pm 0.51$ & $0.001^{*}$ \\
\hline MFT & $8.80 \pm 2.35$ & $11.72 \pm 2.53$ & $0.000^{*}$ & $8.72 \pm 3.35$ & $11.60 \pm 4.04$ & $0.000^{*}$ \\
\hline K-MBI & $47.56 \pm 6.63$ & $52.64 \pm 6.97$ & $0.000^{*}$ & $46.36 \pm 13.82$ & $51.28 \pm 15.14$ & $0.000^{*}$ \\
\hline
\end{tabular}

Values are presented as mean \pm standard deviation.

RCT, combining robot-assisted game training with conventional upper extremity rehabilitation training; OCT, only conventional upper extremity rehabilitation training; MMT, Manual Muscle Test; MFT, Manual Function Test; K-MBI, Korean version of Modified Barthel Index.

${ }^{*} \mathrm{p}<0.05$ by Wilcoxon signed-rank test. 
Table 3. Comparison of the training effects between two groups

\begin{tabular}{lccc}
\hline & $\begin{array}{c}\text { RCT } \\
(\mathbf{n = 2 5})\end{array}$ & $\begin{array}{c}\text { OCT } \\
(\mathbf{n = 2 5})\end{array}$ & p-value \\
\hline$\Delta$ MMT & & & \\
\hline Shoulder adduction & $0.44 \pm 0.71$ & $0.48 \pm 0.51$ & 0.472 \\
\hline Shoulder abduction & $0.48 \pm 0.65$ & $0.52 \pm 0.51$ & 0.588 \\
\hline Elbow flexion & $0.76 \pm 0.78$ & $0.56 \pm 0.58$ & 0.418 \\
\hline Elbow extension & $0.52 \pm 0.71$ & $0.48 \pm 0.65$ & 0.736 \\
\hline Wrist flexion & $0.40 \pm 0.91$ & $0.88 \pm 0.60$ & $0.041^{*}$ \\
\hline Wrist extension & $0.52 \pm 0.71$ & $0.56 \pm 0.51$ & 0.930 \\
\hline Grasp & $0.44 \pm 0.65$ & $0.48 \pm 0.51$ & 0.774 \\
\hline MFT & $2.92 \pm 2.34$ & $2.88 \pm 2.54$ & 0.992 \\
\hline K-MBI & $5.08 \pm 2.61$ & $4.92 \pm 5.37$ & 0.170 \\
\hline
\end{tabular}

Values are presented as mean \pm standard deviation.

RCT, combining robot-assisted game training with conventional upper extremity rehabilitation training; OCT, only conventional upper extremity rehabilitation training; $\triangle \mathrm{MMT}$, change of Manual Muscle Test score from baseline to end of 2-week training; $\triangle \mathrm{MFT}$, change of Manual Function Test score from baseline to end of 2 -week training; $\triangle \mathrm{K}$-MBI, change of Korean version of Modified Barthel Index score from baseline to end of 2-week training.

*p $<0.05$ by Mann-Whitney U-test.

groups in the adduction and abduction of the shoulder joints, flexion and extension of the elbow joints, extension of the wrist joints, and hand grip motion evaluated by MMT, MFT, and K-MBI (Table 3). In the OCT group's $\mathrm{MMT}$, the training effect on flexion of the wrist joints was better than that of the RCT group $(\mathrm{p}<0.05)$ (Table 3$)$.

In a survey of the two groups, the RCT group's degree of satisfaction was $4.12 \pm 0.97$ points and that of OCT was $3.24 \pm 1.01$, with the RCT group having a significantly higher degree of satisfaction $(\mathrm{p}<0.05)$. There was no report of side-effects according due to robot-assisted game training, such as a fall, shoulder or elbow joint pain, or headache.

\section{DISCUSSION}

About $80 \%$ of patients showed total or partial dependence in activities of daily living at 3 weeks after stroke and about $30 \%$ remained so at 6 months to 5 years after [18]. Therefore, immediately after stroke, the main goal is to restore function as soon as possible so that the patient can achieve the best quality of life [19]. Various classic methods to improve stroke patients' muscle strength have been developed, including a neural development technique proposed by Bobath, Rood's technique, which improves muscle strength through stimulation of the superficial skin, Brunnstrom's technique through muscular synergist action, and Kabat's functional improvement through proprioceptive neuromuscular facilitation [2]. However, these various classic rehabilitation treatments have some problems such as repetition of the same motions for a long time, therapists differing opinions on treatment methods and capabilities, and patients becoming bored with treatment [8]. Therefore, recently, new treatment methods, such as robots, games, virtual reality treatments, constraint-induced movement training (CIMT), were introduced [9,20-22]. Among these, robotassisted rehabilitation treatment has numerous benefits and has been developed and used in recent decades $[5,6]$.

Various studies have evaluated robot-assisted rehabilitation treatments. Outcomes of robot-assisted rehabilitation treatments have been variable, depending on the type of robots, study design and the characteristics of patients. Masiero et al. [23] reported that additional robotassisted upper extremity rehabilitation treatment showed greater reductions in motor impairment and improvements in functional abilities after acute stroke. Masiero et al. [16] reported that standard therapy (65\% of exercise time) associated with robotic training ( $35 \%$ of exercise time) had similar improvement in motor, dexterity and activities of daily living compared with conventional rehabilitation treatment. Taveggia et al. [24] reported that rehabilitation treatment with an exoskeleton upper extremity rehabilitation robot ArmeoSpring (Hocoma Inc., Zurich, Switzerland), which allows the pivotal movements of shoulder, elbow and wrist joints in a three dimensional virtual environment was as effective as traditional rehabilitation at the end of 6-week treatment.

Multiple researchers have reported that robot-assisted rehabilitation treatment enhanced upper limb mobility and muscle strength, although its effect on improvements in the activities of daily living was restricted $[5,25,26]$. This is because existing upper-limb robot-assisted rehabilitation treatment was confined to the proximal part of the upper limbs. For functional improvement, coordination between the proximal and distal parts is essential $[27,28]$. Our results showed improvements of motor and 
daily functions in RCT groups, even though the upper extremity rehabilitation robot used in this study limited joint motions of distal parts of upper limb. Perhaps this was because patients in the RCT group performed one daily session of robot-assisted game training plus one daily session of conventional upper extremity rehabilitation training. Patients in the RCT group conducted selftraining after instruction by a therapist. Both groups did not significantly differ in most of MMT, MFT, and K-MBI, suggesting the effects of RCT were similar to those of OCT.

In total, $50 \%$ of stroke patients experience depression and this is considered to result from mental stress about their disability [2]. Such depression may lead to decreased socialization and participation in rehabilitation treatment of stroke patients [2]. The game programs induce pleasure and interest in the rehabilitation therapy, thus acting positively to promote motor learning. The games also inspire, motivate, and trigger pleasure and interest in rehabilitation by utilizing the player's intrinsic sense of competition and desire for interaction, thereby promoting learning movements $[10,29]$. In the present study, the patients in the RCT group expressed greater satisfaction than those in the OCT group in the survey on degree of satisfaction. However, the questionnaire about satisfaction had only a single question. Therefore, additional systematic and appropriate research on the degree of patient satisfaction are considered necessary.

The limitations of this study were that the number of subjects was small and the period of research was short. Additional research to complement these points is considered necessary. Despite such limitations, this study is meaningful in that it is to determine the effects of game training in upper extremity rehabilitation robot Neuro-X plus conventional upper extremity rehabilitation training on upper extremity motor and daily functions compared with those of conventional upper extremity rehabilitation training in stroke patients, as well as examined their degree of satisfaction with the two training methods.

In conclusion, robot-assisted game training can induce patients to perform accurate motions repeatedly; satisfy the patients in training sessions by inducing their interest; can be implemented safely under a therapist's supervision; and can lighten therapists' workloads. The present study showed that RCT provided similar improvement in upper extremity motor and daily function as OCT did. And the patients in the RCT group were more satisfied than those in the OCT group. Our result suggests that RCT could be a useful method for upper extremity training in stroke patient.

\section{CONFLICT OF INTEREST}

No potential conflict of interest relevant to this article was reported.

\section{ACKNOWLEDGMENTS}

This study was promoted as part of the spread business of market creation type robots of the Ministry of Trade, Industry and Energy and was carried out by rehabilitation robots supplied from the Ministry of Health \& Welfare and the National Rehabilitation Center in 2013.

\section{REFERENCES}

1. Harvey RL, Roth EJ, Yu DT, Celnick P. Stroke syndromes. In: Braddon RL, editor. Physical medicine and rehabilitation. 4th ed. Philadelphia: Saunders; 2011. p. 1178-84.

2. Stein J, Brandstater ME. Stroke rehabilitation. In: Frontera WR, DeLisa JA, Gans BM, Walsh NE, Robinson LR, editors. Physical medicine and rehabilitation: principles and practice. 5th ed. Philadelphia: Lippincott Williams \& Wilkins; 2010.

3. Nichols-Larsen DS, Clark PC, Zeringue A, Greenspan A, Blanton S. Factors influencing stroke survivors' quality of life during subacute recovery. Stroke 2005; 36:1480-4.

4. Loureiro RC, Harwin WS, Nagai K, Johnson M. Advances in upper limb stroke rehabilitation: a technology push. Med Biol Eng Comput 2011;49:1103-18.

5. Prange GB, Jannink MJ, Groothuis-Oudshoorn CG, Hermens HJ, Ijzerman MJ. Systematic review of the effect of robot-aided therapy on recovery of the hemiparetic arm after stroke. J Rehabil Res Dev 2006;43:17184.

6. Masiero S, Carraro E, Ferraro C, Gallina P, Rossi A, Rosati G. Upper limb rehabilitation robotics after stroke: a perspective from the University of Padua, Italy. J Rehabil Med 2009;41:981-5.

7. Sivan M, O'Connor RJ, Makower S, Levesley M, Bhakta 
B. Systematic review of outcome measures used in the evaluation of robot-assisted upper limb exercise in stroke. J Rehabil Med 2011;43:181-9.

8. Chang YJ, Chen SF, Huang JD. A Kinect-based system for physical rehabilitation: a pilot study for young adults with motor disabilities. Res Dev Disabil 2011; 32:2566-70.

9. Yong Joo L, Soon Yin T, Xu D, Thia E, Pei Fen C, Kuah $\mathrm{CW}$, et al. A feasibility study using interactive commercial off-the-shelf computer gaming in upper limb rehabilitation in patients after stroke. J Rehabil Med 2010;42:437-41.

10. Saposnik G, Teasell R, Mamdani M, Hall J, McIlroy W, Cheung D, et al. Effectiveness of virtual reality using Wii gaming technology in stroke rehabilitation: a pilot randomized clinical trial and proof of principle. Stroke 2010;41:1477-84.

11. Mouawad MR, Doust CG, Max MD, McNulty PA. Wiibased movement therapy to promote improved upper extremity function post-stroke: a pilot study. J Rehabil Med 2011;43:527-33.

12. Hurkmans HL, Ribbers GM, Streur-Kranenburg MF, Stam HJ, van den Berg-Emons RJ. Energy expenditure in chronic stroke patients playing Wii Sports: a pilot study. J Neuroeng Rehabil 2011;8:38.

13. Yavuzer G, Senel A, Atay MB, Stam HJ. "Playstation eyetoy games" improve upper extremity-related motor functioning in subacute stroke: a randomized controlled clinical trial. Eur J Phys Rehabil Med 2008; 44:237-44

14. Sale P, Mazzoleni S, Lombardi V, Galafate D, Massimiani MP, Posteraro F, et al. Recovery of hand function with robot-assisted therapy in acute stroke patients: a randomized-controlled trial. Int J Rehabil Res 2014;37: 236-42.

15. Fasoli SE, Krebs HI, Stein J, Frontera WR, Hogan N. Effects of robotic therapy on motor impairment and recovery in chronic stroke. Arch Phys Med Rehabil 2003; 84:477-82.

16. Masiero S, Armani M, Ferlini G, Rosati G, Rossi A. Randomized trial of a robotic assistive device for the upper extremity during early inpatient stroke rehabilitation. Neurorehabil Neural Repair 2014;28:377-86.

17. Miyamoto S, Kondo T, Suzukamo Y, Michimata A, Izumi S. Reliability and validity of the Manual Function Test in patients with stroke. Am J Phys Med Rehabil
2009;88:247-55.

18. Shah S, Vanclay F, Cooper B. Efficiency, effectiveness, and duration of stroke rehabilitation. Stroke 1990;21: 241-6.

19. Langhorne P, Pollock A; Stroke Unit Trialists' Collaboration. What are the components of effective stroke unit care? Age Ageing 2002;31:365-71.

20. Chang WH, Kim YH. Robot-assisted Therapy in Stroke Rehabilitation. J Stroke 2013;15:174-81.

21. Saposnik G, Levin M; Outcome Research Canada (SORCan) Working Group. Virtual reality in stroke rehabilitation: a meta-analysis and implications for clinicians. Stroke 2011;42:1380-6.

22. Miltner WH, Bauder H, Sommer M, Dettmers C, Taub E. Effects of constraint-induced movement therapy on patients with chronic motor deficits after stroke: a replication. Stroke 1999;30:586-92.

23. Masiero S, Celia A, Rosati G, Armani M. Roboticassisted rehabilitation of the upper limb after acute stroke. Arch Phys Med Rehabil 2007;88:142-9.

24. Taveggia G, Borboni A, Salvi L, Mule C, Fogliaresi S, Villafane JH, et al. Efficacy of robot-assisted rehabilitation for the functional recovery of the upper limb in post-stroke patients: a randomized controlled study. Eur J Phys Rehabil Med 2016;52:767-73.

25. Mehrholz J, Hadrich A, Platz T, Kugler J, Pohl M. Electromechanical and robot-assisted arm training for improving generic activities of daily living, arm function, and arm muscle strength after stroke. Cochrane Database Syst Rev 2012:CD006876.

26. Kwakkel G, Kollen BJ, Krebs HI. Effects of robot-assisted therapy on upper limb recovery after stroke: a systematic review. Neurorehabil Neural Repair 2008;22: 111-21.

27. Oujamaa L, Relave I, Froger J, Mottet D, Pelissier JY. Rehabilitation of arm function after stroke. Literature review. Ann Phys Rehabil Med 2009;52:269-93.

28. Timmermans AA, Seelen HA, Willmann RD, Kingma $\mathrm{H}$. Technology-assisted training of arm-hand skills in stroke: concepts on reacquisition of motor control and therapist guidelines for rehabilitation technology design. J Neuroeng Rehabil 2009;6:1.

29. Edmans J, Gladman J, Hilton D, Walker M, Sunderland A, Cobb S, et al. Clinical evaluation of a non-immersive virtual environment in stroke rehabilitation. Clin Rehabil 2009;23:106-16. 